\title{
Somatic Gene Therapy, Paradigm Shift or Pandora's Box
}

\author{
A perspective on gene therapy
}

MARK LAWLER

Department of Haematology, St Patrick Dun Research Labs, St James's Hospital and Trinity

College Dublin, James's Street, Dublin 8, Ireland

\section{DEFINITIONS}

Gene therapy may be defined as the introduction of genetic material into the cells of a patient in an effort to help cure the disease either by producing a gene product which is missing or in reduced amounts in the patient due to a genetic mutation in the individual (eg Factor VIII protein for haemophilia) or by introduction of new genetic material which either directly or indirectly will help to combat the disease (eg genetic vaccination). Therapeutic genes are delivered using a carrier (called a vector) which may be a non functional viral vector or by using non viral vector approaches such as liposomes or other carrier molecules. All gene therapy protocols involve the introduction of genetic material into cells that have a finite life span such as blood cells, liver cells etc (termed somatic tissue), thus the introduced gene is not passed on to the next generation. This type of gene therapy is known as somatic gene therapy and is in contrast to the concept of germ line gene therapy (which would involve a gene being introduced into sperm or ova so that the gene could be inherited by the children of the patient). Germ line gene therapy is subject to an international moratorium.

\section{Somatic gene therapy}

Introduction of a gene into a specific tissue or tissues to provide therapeutic benefit to the patient. 


\section{Germ line gene therapy}

Introduction of genetic material into the egg cells or sperm cells of an individual such that the gene will also be passed on to the next generation.

\section{Ex vivo gene therapy}

Collection of the patient's cells, introduction of therapeutic genetic material into these cells and reintroduction of these cells into the patient.

\section{In vivo gene therapy}

Direct injection of therapeutic gene to the relevant tissue via a vector.

The promise of gene therapy lies in its proposed ability to treat the causes of disease rather than the symptoms. The first decade of gene therapy has been somewhat of a "roller coaster" ride, with early excitement of the potential of this approach being tempered somewhat by disappointing clinical results. Recently, however, improvements in vector construction and vector delivery to the appropriate tissue has led to better pre clinical and clinical results.

\section{INTRODUCTION}

Somatic gene therapy involves the amelioration of a disease by introduction of genetic material with therapeutic potential into a somatic tissue. It is only in the last few years that gene transfer could be contemplated in the clinic. Advances have been made which allow the transfer and stable existence of genetic material in a foreign host. Gene therapy has benefited from (A) the gene transfer and expression techniques of molecular genetics; (B) the natural ability of retroviruses to infect foreign replicating cells and stably integrate their genetic material into the host genome and $(\mathrm{C})$ the fact that Bone Marrow Transplantation (BMT) provides a straightforward delivery of in vitro manipulated material into the blood stream. Thus a retrovirus can be engineered to contain an appropriate segment of DNA and ex vivo transduction of haemopoietic cells allows subsequent introduction of foreign material into the host by the BMT route.

There are a variety of gene delivery systems currently available and some systems may be more useful in targeting particular tissues. Retroviruses were the initial vehicles of choice but many studies have made use of adenoviruses or herpes viruses and new developments such as the use of adeno associated virus and non viral delivery systems have great potential. These systems together with the early work in animal studies have been crucial in bringing gene therapy protocols to the clinic. While much of the 
earlier preclinical gene transfer work has concentrated on murine models, it has become clear that use of large animal models including sheep, dogs, pigs and monkeys may be more relevant as the final preclinical step before proceeding to human trials.

While a large number of single gene defects are candidates for a gene therapy protocol and several, including Adenosine Deaminanse (ADA) ${ }^{1}$ and Cystic Fibrosis $(C F)^{2,3}$ have been treated with such protocols it is the realisation that gene therapy has potential in acquired disease which is perhaps the most interesting. The ability to tackle malignant disease either directly through the introduction of genes such as Tumour Necrosis Factor (TNF) or indirectly by introducing immune stimulatory genes may have enormous clinical applications. Gene therapy for malignant diseases will be discussed in Chapter 5. The use of immunostimulatory molecules may also yield dividends in the fight against AIDS. Protocols for cardiovascular disease using either vector or antisense based approaches have shown promise $^{4,5}$ while gene therapy protocols for neurodegenerative disorders are also being developed and applied ${ }^{6,7}$.

\subsection{Vectors and target cells}

Retroviruses have been the most extensively used vectors to date particularly due to the fact that the therapeutic gene is integrated into the host genome. Haemopoietic cells are probably the easiest to target due to the well characterised hierarchical structure of the haemopoietic system. Thus many of the clinical protocols have involved the introduction of a new gene into haemopoetic cells. A second important advantage is that ex vivo gene transfer can be performed - haemopoietic cells can be taken from the individual, infected and then re-introduced to the individual by BMT. Ex vivo gene transfer is very efficient as cells can be externally stimulated to allow higher infection rates with retroviral constructs. The other important advantage of the haemopoietic system is the ability to target stem cells as well as lineage specific cells.

Other targets for retroviral vectors include skin fibroblasts where retroviral infection rates of $50 \%$ have been reported and several clinically important genes including ADA, purine nucleoside phosphorylase (PNP) and Factor IX have been successfully transferred. The skin may be a highly important target for delivery of therapeutic genes - collagen beads with genetically modified fibroblasts secreting Factor IX have been produced and using skin keratinocytes, therapeutic genes could be delivered directly to the circulation.

The major disadvantage of retroviral vectors is their inability to infect non dividing cells. The other major disadvantages relate to the safety aspects 
of retroviruses. It is necessary to do stringent testing of cell lines for potential replication competent retroviruses. Clearly insertional mutagenesis and the worry that retroviral activation of cellular oncogenes has occurred in murine systems must also be considered. Finally the fact that the maximum size of DNA which can be efficiently packaged and transduced is approximately $7 \mathrm{~kb}$ limits their potential for certain diseases. Specialised packaging cell lines capable of producing high titres of replication deficient recombinant virus free of any wild type viral contaminants have been produced. However, outbreaks have been reported presumably due to contaminating replication competent viruses. The prevention of such outbreaks must of course be avoided. This has been aided by the development of better modifications to packaging lines and by more stringent monitoring of the products of such lines.

\subsection{Adenoviruses}

Adenoviruses are potentially more useful in in vivo gene therapy. They can infect non dividing cells, larger sizes of exogenous DNA ( $>30 \mathrm{kbs}$ ) can be incorporated and high titres of the virus can be produced which is of great importance for in vivo applications. The virus particle itself is relatively stable. One worry is the presence of similar sequences in the human genome which could combine with the inserted sequences leading to development of malignancy. Replication deficient adenovirus can be generated and propagated by growth in cells engineered to express the El replication region, thus allowing the development of adenovirus vectors expressing large amounts of a foreign gene product in vitro. Pre clinical studies have indicated that efficient transduction in vivo occurs and gene expression can be seen for significant periods post transduction. Adenoviruses have been used to deliver the Cystic Fibrosis gene to airway epithelium and the ability of adenoviral vectors to target brain, liver and muscle cells have indicated that adenoviruses may become major vectors in clinical protocols. However adenovirus vectors tend to be recognised by the host's immune system and so need to be modified greatly to avoid immunisation and clearance of the therapeutic vector.

\subsection{Other viral vectors}

The most important of these include adeno-associated virus (where their ability to integrate at a particular locus on chromosome 19 might allow controlled precise expression of any inserted gene), and herpes simplex virus which could be highly important in neurodegenerative disorders ${ }^{8}$. Adeno associated viruses are very simple viruses to produce and their broad host 
range in conjunction with adenovirus make them useful vector systems. The scope of such systems is enormous and will mean that even vector systems that we might consider enemies (such as the Human Immunodeficiency virus) may prove to be friends at the gene therapy level due to their target cell specificity'.

\section{$2.4 \quad$ Non viral delivery systems}

The majority of the work currently reported in the literature has focused on the use of viral vectors to deliver the desired gene product to its target cell or tissue; however there is a growing body of evidence that non viral methods may be useful in the potential treatment of several single or polygenic disorders. While a variety of methods including the direct injection of naked DNA into muscle cells, arterial walls or the heart itself have been shown to be feasible, an approach which would have general implications for a variety of diseases has involved the delivery of DNA protein complexes to a specific cell via a receptor molecule intermediate. The main advantages associated with non viral methods are (A) their ability to deliver large transgenes (up to $50 \mathrm{~kb}$ ) to their target; (B) their ability to target different receptors; (C) a safer approach since viral integration is avoided. This receptor mediated delivery system has been used to deliver the low density lipoprotein receptor (LDLr) to the circulation of Watanebe rats and the lowering of subsequent total serum cholesterol by this treatment provide a good animal model for a gene therapy protocol for hypercholesteremia ${ }^{10}$. Recent studies are also indicating that it may be much easier than we at first believed to get DNA into the cell through the use of anitisense approaches or "naked DNA " injection".

\section{$2.5 \quad$ Tissue specific gene delivery}

While the majority of pre-clinical work has focused on delivery of therapeutic genes to the haemopoietic system, there have been many attempts to target other tissues also. In the liver, the primary candidates would be the hepatocyte but while transducing hepatocytes is relatively straightforward, the transplantation of such hepatocytes has proven problematic. This may be overcome by using methods for ectopically grafting hepatocytes or by in vivo transduction by retrovirus. Delivery of gene products to the circulation will also be important for a wide variety of diseases and so a method for efficient delivery of transgenes is necessary. Retroviral transduction of keratinocytes has been achieved but expression of exogenous genes was short lived. Both factor IX and growth hormone can be expressed in myoblast cell lines using vectors which contained non viral 
control sequences and these cells can be successfully re-implanted into animals $^{12}$, while correction of the lysosomal storage defect in $\beta$ glucuronidase deficient mice (the animal model for Sly syndrome, a human mucopolysaccharide disorder) has been achieved by autologous transplantation following retroviral infection of fibroblasts ${ }^{13}$.

\section{WHERE DID GENE THERAPY BEGIN?}

The development of gene therapy as we know it today has resulted from two significant advances in science and medicine in the 1960s and 70s - the advances in cellular and transplantation biology leading to effective bone marrow transplant treatment for leukaemia and the advances in molecular biology and genetic engineering leading to the cloning of therapeutic proteins for the treatment of human disease.

\subsection{Tumour infiltrating lymphocytes}

The Neo ${ }^{r}$ TIL protocol, the first approved protocol for gene transfer in humans, was initiated at NIH in 1988 and provided the springboard for the subsequent ADA protocol which will be discussed in section 2. The basis of this work was the finding that there is a class of cell called a tumour infiltrating lymphocyte (TIL) which has potential activity against a patient's own cancer. These cells can be isolated from a patient and expanded using interleukin 2. These TILs are classic cytotoxic T cells and injection of IL2 expanded TILs into cancer bearing mice leads to tumour regression. The next step involved establishing their efficacy in patients. TILs from end stage melanoma patients were collected from tumour nodules, expanded with IL2 and reintroduced intravenously into the patient. 55\% of the group responded well to this therapy. Subsequently it was decided to look at the feasibility of marking TILs prior to re-introduction into the patient. The neophosphotransferase resistance (neo') gene was chosen as a marker gene and a clinical trial of end stage melanoma patients was initiated after many reviews and delays to ensure the safety of the technique. On May 22 1989, the first patient was treated and a series of 5 patients were reported on in the New England Journal of Medicine in 1990. While this protocol was not a therapeutic protocol but a simple "marking" protocol, it provided the impetus for the second stage in gene transfer in humans, the transfer of a gene with therapeutic potential ${ }^{14,15}$. 


\subsection{ADA deficiency}

Initially $\beta$ thalassemia was thought to be the disease of choice for genetic manipulation but by 1984 it became clear that the haemoglobinopathies were too complex for early attempts at gene therapy. One of the first criteria for a suitable gene therapy model is for the corrected cells to have a growth advantage in the patient. Obvious candidates would be mutations in DNA metabolism as corrected cells would show more efficient cell division. Possibilities focused on the sequential steps in purine metabolism involving the genes Adenosine Deaminase (ADA), Purine Nucleosyl Phosphorylase (PNP) and Hypoxanthine guanine PhosphoRibosyl Transferase (HPRT) which are all known to cause diseases due to enzyme deficiency. ADA deficiency leads to an immune deficiency syndrome and makes these patients very susceptible to infection, thus necessitating that they live in a carefully controlled environment. Evidence from BMT of ADA patients indicated that $\mathrm{T}$ cells from the donor easily outgrow ADA deficient cells therefore ADA was chosen as the initial model system for gene therapy. In ADA deficiency there is a severe depletion of the number and activity of $T$ cells while there is also a debilitating effect on B cells. Thus cellular and humoral activity is severely compromised and death usually ensues from infection in the first 2 years of life. As matched BMT has been shown to cure $\mathrm{ADA}$ it is clear that replacement of the abnormal $\mathrm{T}$ cells with normal $\mathrm{T}$ cells is sufficient to cure the disease. The lack of success with mismatched BMT means that alternative strategies need to be attempted. The finding that individuals can have as little as $5 \%$ or as much as 50 times the standard levels of ADA are also important factors for a preliminary gene therapy protocol as the level of expression need not be under stringent control.

Thus on September 14th 1990, the first child was treated for this disease by gene therapy. Blood was taken from the patient, a four year old girl with no immune function ${ }^{16}$. Red cells were given back by leukophoresis and mononuclear cells isolated by Ficoll centrifugation. These cells were grown in tissue culture, stimulated with IL2 and infected with a third generation retrovirus containing the ADA gene and a neo marker gene. The girl received 8 infusions in an 11 month period of the transduced cells and was also on weekly injections of Polyethylene glycol (PEG) ADA. PCR showed gene corrected $\mathrm{T}$ cells $(20-25 \%)$ in the mononuclear cell population. Clinical condition had improved so subsequently she received maintenance gene therapy infusions at 6 month intervals. A second patient (a 9 year old girl) received 11 infusions of gene corrected autologous $\mathrm{T}$ cells from January 1991. Results in both patients were encouraging with both attending school and showing no side effects and average number of infections for children of their age. ADA levels were at $25 \%$ of normal and it was estimated that the 
half life of gene corrected $\mathrm{T}$ cells could be as high as $2-3$ years whereas abnormal $\mathrm{T}$ cells in the patient have a half life of approximately 2-6 months. Subsequently a second study was performed in Europe which also indicated long term expression of the gene in transfected $\mathrm{T}$ cells reintroduced into the patient ${ }^{17}$. However these studies were complicated by the fact that these patients were receiving PEG ADA also and it was unclear if sufficient long term expression of ADA in gene corrected $T$ cells could significantly alter the phenotype. Removal of the PEG - ADA led to a reduction in ADA expression, clearly indicating that while the potential for gene therapy was there, improvements in efficient delivery and gene expression were required $^{18}$.

Current work is focusing on the delivery of gene-corrected stem cells so that educable $\mathrm{T}$ cells might be achieved with continual ADA production. Here CD 34 cells (early progenitor cells) are used in combination with "adult cells" to preserve potential stem cell repopulation. In order to monitor stem cell repopulation, different vectors are being used for stem cells and differentiated cells and pre treatment with granulocyte colony stimulating factor (G-CSF) would allow stimulation of the circulation of stem cells normally present in bone marrow. Introduction of the ADA gene into primitive cells is being attempted by either manipulation of the microenvironment through the use of molecules such as fibronectin or by using other sources of stem cells such as cord blood. In this regard recent work has indicated that the retroviral infection efficiency may be superior in cord blood stem cells than in adult bone marrow. A number of children have been treated with constructs that have been introduced into umbilical cord cells. Despite an improvement in clinical symptoms and treatment of other children using these protocols, there is no evidence to date of a patient with ADA deficiency having long term cure of their disease by gene therapy ${ }^{1}$.

\subsection{Other enzyme deficiencies}

Other metabolic disorders are also good candidates for gene therapy based approaches, as $5-25 \%$ of normal enzyme activity will normally suffice for protection from clinical disease in disorders such as Haemophilia B. Several metabolic disorders are caused by absence of specific lysosomal enzymes that degrade specific compounds. The inability to degrade these compounds can lead to organ dysfunction, both visceral and in the CNS. Gaucher disease, a deficiency of $\beta$ glucocerebrosidase is treatable with enzyme replacement therapy and BMT, thus showing the potential for a gene therapy directed approach. Clinical trials involving the introduction of retroviral vectors containing the $\beta$ glucocerebrosidase cDNA into BM or stem cells are underway ${ }^{19}$. Animal studies in Sly syndrome where $\beta$ 
glucorinidase deficiency results in accumulation of sulphated glycoaminoglycans has indicated that autologous fibroblasts transfected with appropriate vectors and transplanted in mice can correct the lysosomal storage problems and serve as a model for the human situation ${ }^{13,20}$.

Recent studies in the haemophilia B dog model also suggest that gene therapy approaches may be useful in the treatment of this disorder in humans. A single intraportal vein injection of a recombinant adenoassociated virus (rAAV) vector encoding canine factor IX (cFIX) cDNA under the control of a liver-specific enhancer/promoter led to long-term correction of the bleeding disorder in haemophilia B dogs. Both whole-blood clotting time (WBCT) and activated partial thromboplastin time (aPTT) of the treated dogs have been greatly decreased since the treatment supporting the feasibility of using AAV-based vectors for liver-targeted gene therapy of genetic diseases ${ }^{21}$.

\subsection{Cystic fibrosis}

While adenosine deaminase deficiency has perhaps proved the most amenable disease to gene therapy, it is a rare genetic disorder. Cystic fibrosis (CF) however is a lethal inherited disorder which affects approximately 1 in 2,000 caucasians. Since the cloning of the cystic fibrosis transmembrane conductance regulator (CFTR) gene in 1989, effective and safe treatment of the underlying defect by gene therapy has become one of the principle aims of researchers in this area. Several important breakthroughs at the molecular level have meant that this possibility may be imminent, even though a fuller understanding of the molecular defect causing $\mathrm{CF}$ is probably required for long term amelioration of the genetic defect.

The first important discovery was that the cystic fibrosis could be corrected in vitro by retrovirus mediated gene transfer. Subsequent work indicated that the human CFTR gene could be directed to the lung epithelium in cotton rats using a replication deficient adenovirus vector, a vector known to infect the respiratory epithelium and capable of transferring recombinant genes into non proliferating cells. A second important development in this area was the production of homozygous CFTR deficient mice. These mice were produced by "knock out" homologous recombination and represent the first authentic animal model for CF. These mice allowed the re-introduction and expression of CFTR in a variety of cell types and thus provide vital knowledge for the application of somatic gene therapy for this disease.

The central question for CF gene therapy is what level of CFTR expression is required to achieve and maintain normal function. It may be as 
little as $10 \%$. This would be important as it may indicate that $100 \%$ correction of epithelial cells is not necessary to repair the chloride transport defect that is crucial to the pathogenesis of the disease. A second question relates to the type of cell which must be corrected and at what stage in development this should be performed. Although the epithelia of the lung is the major site of pathology and morbidity associated with the disease, very low expression levels of CFTR are present in adults, whereas high levels of CFTR mRNA can be detected in foetal tissue. This may have implications for early intervention in this disorder. Currently, a variety of protocols have been approved for gene therapy in CF using adenovirus delivery systems to the pulmonary epithelium. While initial results proved promising a worrying aspect occurred during dose escalation studies associated with the trial. A woman who had received a high titre of gene manipulated adenovirus developed fever and lung inflammation which prompted re-evaluation and lowering of the dose of modified virus. A second trial looked at the efficacy of gene transfer to CF patients using a liposomal DNA complex spray administered through the nose. This non viral delivery route may be a more suitable route for administration of normal CFTR to the appropriate tissue. Recently a third generation adenoviral vector containing recombinant human cystic fibrosis transmembrane conductance regulator (CFTR) gene was delivered by bronchoscope in escalating doses to the conducting airway of 11 volunteers with cystic fibrosis. These results demonstrate that gene transfer to epithelium of the lower respiratory tract can be achieved in humans with adenoviral vectors but that efficiency is low and of short duration in the native $\mathrm{CF}$ airway ${ }^{22}$.

\subsection{Neurological disease}

While gene therapy for the disorders already discussed usually involves the delivery of the ameliorating gene to a specific cell type, it may appear initially that gene therapy for the nervous system would present more complications due to the wide range of highly differentiated cell types in the central nervous system. Thus any gene therapy protocol would involve a gene targeting system that was suitable for infection of these different cell types. Since most mature neurons do not replicate, the use of retroviral vectors may not be the most suitable in this area. However, the ability to target other cells and use them as surrogate carriers of the corrective gene to the brain may be a more effective method of treating neurological disorders, such as Alzheimer's or Parkinson's disease.

The earliest work in this area concentrated on the modification and transplantation of autologous fibroblasts. Animal studies on Alzheimer's disease indicated that intracerebral grafting of fibroblasts that had been 
genetically modified to produce nerve growth factor prevented the death of cholinergic neurons in the basal forebrain which had been associated with profound cognitive impairment in this animal model ${ }^{23}$. A similar method may also be useful for Parkinson's disease where non neural cells could be genetically modified to produce dopamine or the dopamine precursor L DOPA. While these approaches of indirect gene transfer may prove useful it is also clear that for some disorders the genetic material must be transferred directly to the resident brain cell. This would require the development of neurotropic vectors - the herpes simplex virus is an excellent candidate due to its high infection efficiency in neuronal cells. Herpes simplex viral vectors have been developed by a number of groups and in studying Lesch Nyhan disease it has been shown that functional human HPRT could be introduced into the brains of normal rats by replication competent viral vectors ${ }^{24}$.

While these results are promising, problems of cytotoxicity and poor gene expression with these systems have indicated that these vectors must be optimised for more routine use. Although heterologous genes can be expressed in the brain using different technologies, further studies are now necessary to characterise the long term viability of transferred cells/vectors and their expressed transgenes and their potential for inducing direct or indirect neuropathological changes.

\subsection{Gene therapy for infectious disease}

Two strategies are currently in vogue in the potential treatment of diseases such as AIDS. One involves the use of intracellular immunisation and is designed to render cells resistant to viral replication whereas the second involves the use of genetically modified cells which express viral gene products, thus inducing antiviral cellular immune responses ${ }^{25,26}$. RNA based inhibitors include antisense RNA, ribozymes and RNA decoys have also been used in preclinical studies. Antisense technology involves the production of an artificial molecule which is complementary to the normal sense strand and thus blocks the messenger RNA from subsequent translation. Initial antisense studies showed limited efficacy with transcripts directed against tat, rev, gag or the primer binding site. Tat and Rev are key regulatory gene products which bind to specific regions of the viral RNA termed TAR and RRE and activate transcription of viral genes. Subsequent use of an adeno associated virus construct allowed introduction of antisense to the HIV 1 LTR including the TAR sequence. This antisense resulted in specific down regulation of LTR TAR expression in vitro ${ }^{27}$. Further studies are underway using this approach. 


\section{SAFETY ISSUES ASSOCIATED WITH GENE THERAPY}

The US Recombinant DNA Advisory Committee (RAC) was established in 1975 and in the area of gene therapy has advocated open and public discussion of advanced therapeutic products and protocols. Stringent vetting of proposals is performed and they stress the need for full disclosure of positive and negative results and potential side effects of gene therapy. The US Food and Drug Administration (FDA) have issued a Note for Guidance on the use of human somatic cell therapy and gene therapy in March 1998. The European Commission communication (OJ EC C229/4 issued on 22/07/1998) provides details on human gene therapy and regulations but is currently being revised. The European Agency for the Evaluation of Medicinal Products (EMEA) has recognised the need for consistent regulations in relation to gene therapy. In February 1999 it published a concept paper (CPMP/BWP/2257/98) of its Biotechnology Working Party entitled "Concept paper on the development of a committee for proprietary medicinal products (CPMP) points to consider on human somatic cellular therapy". This has led to the release of 2 discussion papers CPMP/BWP/41450/98 and CPMP/BWP/3088/99 for discussion and consultation which form the basis of current regulation of gene therapy and gene therapy products in Europe.

Nevertheless despite these regulations and proposed regulations in force in Europe and North America, in September 1999, the first death directly attributable to gene therapy occurred. The patient was being treated for an enzyme deficiency called ornithine transcarbamylase deficiency (OTC) as part of a phase 1 clinical study. The patient was in the group that received the highest dose in the trial protocol of an adenoviral vector containing the OTC gene. He developed acute respiratory distress syndrome (ARDS) shortly after the gene therapy infusion and died 2 days later from organ failure. Measurement of cytokine levels indicated that he had systemic inflammatory response syndrome; all erythroid precursor cells were wiped out from his marrow and the vector had gone to other organs besides the $\operatorname{liver}^{28}$. Subsequently the FDA found procedural problems and shut down all 7 clinical trails at Penn's Institute for Human Gene Therapy. Problems related to consent, and the death of two animals in similar preclinical procedure indicates that as in all other therapeutic approaches, full and frank disclosure of any problems should be performed. 


\section{WHERE DO WE GO FROM HERE?}

It is important to realise at this stage that gene therapy is no longer a dream; it has become a reality. Protocols have currently been approved in the US and Europe for a variety of genetic and acquired diseases involving over 300 patients. The initial excitement and preliminary work has of course focused on the genetic disorders. However diseases such as ADA deficiency probably only affect $100-150$ people in the US and even the more common genetic disorders such as the haemoglobinopathies would affect less than 100,000 individuals in the US although they are far more widespread in the Third World. It is the ability of gene therapy to be a viable modality of therapy for acquired genetic disease or diseases with a genetic component that is potentially the most exciting. If gene therapy strategies can be tailored as outlined to treat neuro-degenerative disorders, cardiovascular disease and cancer, then the potential for cure of disease in millions of patients worldwide is within reach. This is why many biotechnology companies which specialised in recombinant DNA technologies in the 80's are now developing active gene therapy programmes in the new millennium. In addition most large pharmaceutical drug companies have gene discovery and gene therapy programmes.

But how close are we in reality? While the approaches outlined are very exciting, there are still major problems to be addressed. The treatments described thus far have in general concentrated on ex vivo gene therapy using viral vectors. The main problem with expanding gene therapy to treat large numbers of patients is the complexity of the current protocols. Ex vivo protocols require the extraction and manipulation of patient specific cells and their re-introduction into the patient. This is probably only feasible in highly specialised institutes although several companies will engineer the gene of interest into patient cells and then return them to the physician for subsequent therapy. Also while initial ADA gene therapy infusions were performed in intensive care, recent treatments have involved more routine operations. Gene therapy is perhaps a natural progression from blood transfusion and bone marrow transplantation, indeed the blood transfusion centres of today may be the gene therapy centres of the future as the need for conventional blood products decreases with the availability of synthetic substitutes. Some transfusion centres already collect stem cells for treatment of leukaemia so their metamorphosis to gene therapy vector centres is not too far-fetched. The recent advances in antisense technology also look promising. 
It is necessary of course to temper our excitement not only with a degree of realism but also with a high regard for the safety aspects of this new therapy. Gene therapy is at a very early stage so we have no ideas of what long term side effects may ensue. The development of tumours in primates due to replication competent retroviral contamination provided a timely warning of the potential hazards of gene therapy and has led to much more rigorous testing of vectors and the development of 3rd and 4th generation vector systems with added safety features. If we think about it, the long term aim of any protocol would be a type of 'genes in a bottle " approach where the gene of interest could be injected just like a pharmaceutical drug. Thus it could be injected intravenously much as insulin is delivered in diabetics. Perhaps the "gene-drug" is on the horizon.

\section{REFERENCES}

1. Parkman R, Weinberg K, Crooks G, Nolta J, Kapoor N, Kohn D, Gene therapy for adenosine deaminase deficiency. Annu Rev Med. 2000;51:33-47

2. Zeitlin PL Cystic fibrosis gene therapy trials and tribulations. Mol Ther. 2000;1(1):5-6.

3. Alton E, Geddes D Cystic fibrosis clinical trials. Adv Drug Deliv Rev. 1998 2;30(1-3):205217.

4. Mann MJ Gene therapy for peripheral arterial disease. Mol Med Today. 2000;6(7):285-91.

5. Raizada MK, Francis SC, Wang H, Gelband CH, Reaves PY, Katovich MJ. Targeting of the renin-angiotensin system by antisense gene therapy: a possible strategy for the longterm control of hypertension. Hypertens. $2000 \mathrm{Apr} ; 18(4): 353-62$.

6. Stedman H, Wilson JM, Finke R, Kleckner AL, Mendell J Phase I clinical trial utilizing gene therapy for limb girdle muscular dystrophy: alpha-, beta-, gamma-, or deltasarcoglycan gene delivered with intramuscular instillations of adeno-associated vectors. Hum Gene Ther. 2000 20;11(5):777-90.

7. Bjorklund A The use of neural stem cells for gene therapy in the central nervous system. Gene Med. 1999;1(3):223-6

8. Latchman DS. Herpes virus vectors for gene therapy in the nervous system. Biochem Soc Trans. 1999;27(6):847-51

9. Costello E, Munoz M, Buetti E, Meylan PR, Diggelmann H, Thali M. Gene transfer into stimulated and unstimulated T lymphocytes by HIV-1-derived lentiviral vectors. Gene Ther. 2000 7(7):596-604 
10. Stein CS, Martins I, Davidson BL Long-term reversal of hypercholesterolemia in low density lipoprotein receptor (LDLR)-deficient mice by adenovirus-mediated LDLR gene transfer combined with CD154 blockade. J Gene Med. 2000;2(1):41-51

11. Restifo NP, Ying H, Hwang L, Leitner WW. The promise of nucleic acid vaccines. Gene Ther. $2000 ; 7: 89-92$.

12. Thompson AR. Gene therapy for the haemophilias. Haemophilia. 2000;6 Suppl 1:115-9

13. Ohashi T, Yokoo T, Iizuka S, Kobayashi H, Sly WS, Eto Y. Reduction of lysosomal storage in murine mucopolysaccharidosis type VII by transplantation of normal and genetically modified macrophages. Blood. 2000;95(11):3631-3

14. Rosenberg SA, Aebersold P, Cornetta K, Kasid A, Morgan RA, Moen R, Karson EM, Lotze MT, Yang JC, Topalian SL, et al Gene transfer into humans--immunotherapy of patients with advanced melanoma, using tumour-infiltrating lymphocytes modified by retroviral gene transduction. $N$ Engl J Med. 1990;323(9):570-8

15. Rosenberg SA. Immunotherapy and gene therapy of cancer. Cancer Res. 1991;51(18 Suppl):5074s-5079

16. Blaese RM, Culver KW, Chang L, Anderson WF, Mullen C, Nienhuis A, Carter C, Dunbar $C$, Leitman S, Berger M, et al. Treatment of severe combined immunodeficiency disease (SCID) due to adenosine deaminase deficiency with $\mathrm{CD} 34+$ selected autologous peripheral blood cells transduced with a human ADA gene. Amendment to clinical research project, Project 90-C-195, January 10, 1992. Hum Gene Ther. 1993;4(4):521-7

17. Bordignon C, Notarangelo LD, Nobili N, Ferrari G, Casorati G, Panina P, Mazzolari E, Maggioni D, Rossi C, Servida P, et al. Gene therapy in peripheral blood lymphocytes and bone marrow for ADA- immunodeficient patients. Science. 1995;270(5235):470-5

18. Anderson WF. Human gene therapy. Nature. 1998;392(6679 Suppl):25-30

19. Barranger JA, Rice EO, Swaney WP Gene transfer approaches to the lysosomal storage disorders. Neurochem Res. 1999;24(4):601-15

20. Sferra TJ, Qu G, McNeely D, Rennard R, Clark KR, Lo WD, Johnson PR. Recombinant adeno-associated virus-mediated correction of lysosomal storage within the central nervous system of the adult mucopolysaccharidosis type VII mouse. Hum Gene Ther. 2000 Mar;11(4):507-19

21. Wang L, Nichols TC, Read MS, Bellinger DA, Verma IM. Sustained expression of therapeutic level of factor IX in hemophilia B dogs by AAV-mediated gene therapy in liver. Mol Ther. 2000;1(2):154-8

22. Zuckerman JB, Robinson CB, McCoy KS, Shell R, Sferra TJ, Chirmule N, Magosin SA, Propert KJ, Brown-Parr EC, Hughes JV, Tazelaar J, Baker C, Goldman MJ, Wilson JM. A phase I study of adenovirus-mediated transfer of the human cystic fibrosis transmembrane conductance regulator gene to a lung segment of individuals with cystic fibrosis. Hum Gene Ther. 1999;10(18):2973-85 
23. Blesch A, Grill RJ, Tuszynski MH Neurotrophin gene therapy in CNS models of trauma and degeneration. Prog Brain Res. 1998;117:473-84

24. Lowenstein PR, Southgate TD, Smith-Arica JR, Smith J, Castro MG. Gene therapy for inherited neurological disorders: towards therapeutic intervention in the Lesch-Nyhan syndrome. Prog Brain Res. 1998;117:485-501

25. Kohn DB, Bauer G, Rice CR, Rothschild JC, Carbonaro DA, Valdez P, Hao Q1, Zhou C, Bahner I, Kearns K, Brody K, Fox S, Haden E, Wilson K, Salata C, Dolan C, Wetter C, Aguilar-Cordova E, Church J. A clinical trial of retroviral-mediated transfer of a revresponsive element decoy gene into $\mathrm{CD} 34(+)$ cells from the bone marrow of human immunodeficiency virus 1-infected children. Blood. 1999;94 (1):368-71.

26. Amado RG, Mitsuyasu RT, Zack JA. Gene therapy for the treatment of AIDS: animal models and human clinical experience. Front Biosci. 1999;4:D468-75

27. Lamothe B, Joshi S. Current developments and future prospects for HIV gene therapy using interfering RNA-based strategies. Front Biosci. 2000;5:D527-55

28. Gene Therapy - a loss of innocence. Nature Medicine 2000; 6 (1) 1 